\title{
Enhancement of spontaneous emission rate and reduction in amplified spontaneous emission threshold in electrodeposited three-dimensional ZnO photonic crystal
}

\author{
Yongchun Zhong, ${ }^{1,2}$ Zhounan Yue, ${ }^{1}$ George K. L. Wong, ${ }^{1}$ Yan Yan Xi, ${ }^{3}$ Yuk Fan Hsu, ${ }^{3}$ \\ Aleksandra B. Djurišić, ${ }^{3}$ Jian-Wen Dong, ${ }^{4}$ Wen-Jie Chen, ${ }^{4}$ and Kam Sing Wong ${ }^{1, a)}$ \\ ${ }^{1}$ Department of Physics, Hong Kong University of Science and Technology, Hong Kong, People's Republic \\ of China \\ ${ }^{2}$ Key Laboratory of Optoelectronic Information and Sensing Technologies of Guangdong Higher Education \\ Institutes, Jinan University, Guangzhou 510632, People's Republic of China \\ ${ }^{3}$ Department of Physics, The University of Hong Kong, Hong Kong, People's Republic of China \\ ${ }^{4}$ State Key Laboratory of Optoelectronic Materials and Technologies, Sun Yat-sen (Zhongshan) University, \\ Guangzhou 510275, People's Republic of China
}

(Received 14 June 2010; accepted 18 September 2010; published online 8 November 2010)

\begin{abstract}
$\mathrm{ZnO}$ photonic crystal (PC) with face-center-cube type structure is fabricated by electrodeposition using holographic lithographically made organic (SU-8) template. Photonic band gap effect (reflection peak and transmission dip in infrared spectral region) is clearly seen. Observation of strong enhancement and blueshift of the emission peak (from 383.8 to $378.8 \mathrm{~nm}$ ), shortening of the exciton photoluminescence lifetime (from 88 to $34 \mathrm{ps}$ ), and reduction in amplified spontaneous emission threshold of $\mathrm{ZnO} \mathrm{PC}$ compared to that of the reference nonstructured electrodeposited $\mathrm{ZnO}$ showed clear evidence of PC structure affecting the $\mathrm{ZnO}$ exciton emission. () 2010 American Institute of Physics. [doi:10.1063/1.3499274]
\end{abstract}

Due to their promising potential for miniaturization and integration of optical devices, photonic crystals (PCs) have been extensively studied since the first proposal in $1987 .{ }^{1} \mathrm{~A}$ number of techniques such as self-assembly, multiphoton polymerization, and holographic lithography (HL) have been used to make PCs. ${ }^{2-4}$ However, to achieve a full photonic band gap, it is necessary to use a high refractive index material. This is typically achieved by infiltration of a high refractive index material in a PC template. ${ }^{5,6}$ Infiltration can be accomplished by different methods, such as chemical vapor deposition (CVD) and atomic layer deposition (ALD). ${ }^{2,5,6}$ However, both of these methods are conformal coating approaches, which will result in an incomplete filling of the template due to pinch-off problem, ${ }^{7,8}$ which can lead to the loss of photonic band gap. ${ }^{8}$ Unlike conformal coating processes such as CVD or ALD, inexpensive solution-based techniques such as electrodeposition ${ }^{9,10}$ and electrophoretic deposition ${ }^{8}$ allow almost complete filling of the template.

Among various materials for infiltration, $\mathrm{ZnO}$ appears to be a very interesting one because of its relative high refractive index $(\mathrm{n} \sim 2.0)$ and its remarkable properties as a semiconductor and transparency in the visible range of the spectra. ${ }^{11}$ Recently, high-quality $\mathrm{ZnO}$ inverted opals grown by different methods $5,6,12$ have been reported. However, the templates used in these previous works were fabricated by self-assembly method, which can only be fabricated facecenter-cube (fcc) and close-packed hexagonal lattice. Here we demonstrate the use of HL to prepare $\mathrm{ZnO}$ PCs. HL is one of the most promising techniques for mass production of large area, defect free one-, two-, and three-dimensional PC structures. ${ }^{13}$ It was proven that umbrellalike multibeam interference could fabricate all bravais lattices, ${ }^{14}$ and various other ordered structures. Recently, we have developed a HL

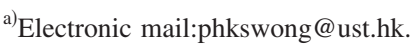

technique using a single-beam and a refracting prism to split and combine the beams to make the desired interference pattern. $^{13,15}$ This single-beam and single-refracting prism HL technique is very simple and stable compared to conventional multibeam and multielements setup.

The templates were fabricated by single beam HL using photoresist (SU-8) deposited on indium tin oxide coated glass substrate, with the details of the procedure and schematic illustration of the setup given in Ref. 13. Figure 1(a) shows the scanning electron microscopy (SEM) image of the organic template. The inset is the cross section of the template. A high quality, defect free fcc-type structure was clearly seen in these SEM images. Figure 1(b) is the simu-

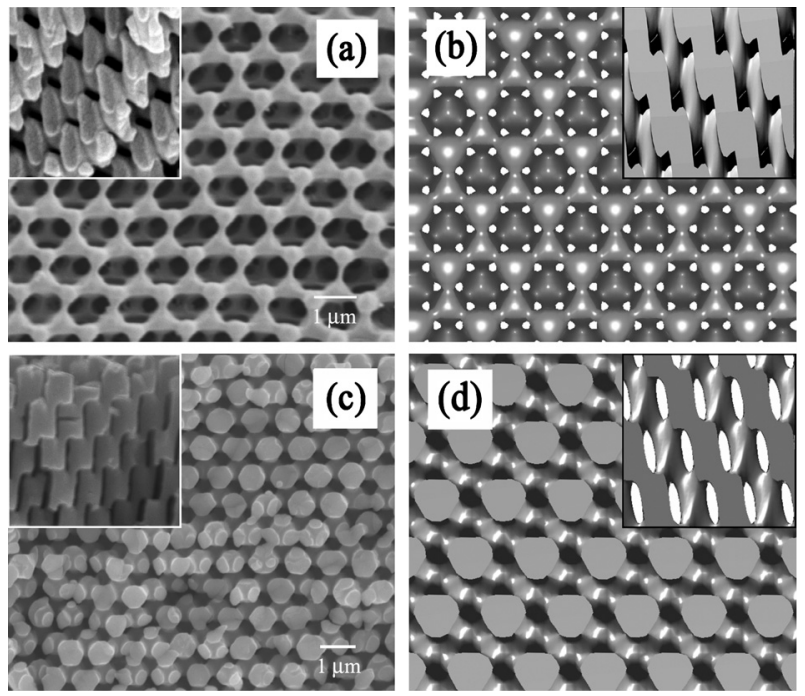

FIG. 1. (a) The SEM images of PC template. The figure inset is the cross section of the sample. (b) The computer simulation of (a). (c) The close-up SEM image of the $\mathrm{ZnO}$ inverse PCs. The inset is the cross section of the sample. (d) shows the computer simulation of (c). 


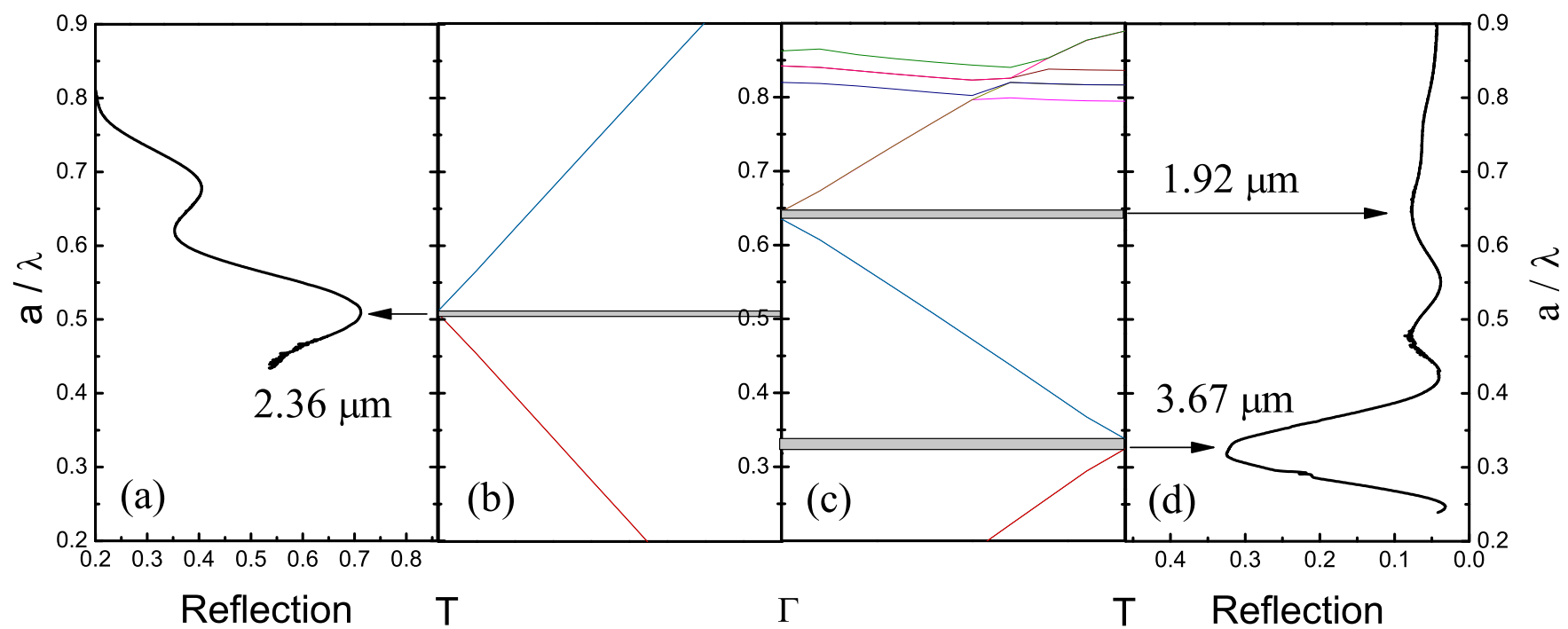

FIG. 2. (Color online) (a) Optical intensity reflection spectrum of the fcc-type photoresist template in Fig. 1(a), showing one pronounced peaks around $2.36 \mu \mathrm{m}$. (b) Corresponding band-structure calculation along the measured direction $(\Gamma-\mathrm{T})$. (c) Corresponding band-structure calculation of the $\mathrm{ZnO}$ inverse PC in Fig. 2(a). The gray shaded area corresponds to the band gap at the measured direction. (d) Optical intensity reflection spectrum of the ZnO inverse PC in Fig. 2(a), showing one pronounced peaks around $3.67 \mu \mathrm{m}$ and two shoulders at 2.60 and $1.92 \mu \mathrm{m}$.

lation of the interference pattern which shows good agreement between the experimental result and simulation.

$\mathrm{ZnO}$ was infiltrated into the HL made organic template using electrodeposition technique. After electrodeposition, the samples were rinsed by deionised water and ethanol respectively, and then annealed at $500{ }^{\circ} \mathrm{C}$ in air for $30 \mathrm{~min}$. to remove the polymer template. Large area and high quality $\mathrm{ZnO}$ microstructure with fcc-type lattice was obtained. The uniform area is about $10 \mathrm{~mm}^{2}$. Figure 1(c) shows the close-up of the $\mathrm{ZnO}$ inverse structure. The inset is the cross section of the sample. Complete filling of the SU-8 template pore network by $\mathrm{ZnO}$ without any discontinuity is clearly seen. The simulation of the interference pattern is shown in the Fig. 1(d). All the SEM images are in good agreement with the simulation.

Figures 2(a) and 2(d) show the reflection spectra of the SU-8 template and $\mathrm{ZnO}$ inverse $\mathrm{PC}$, respectively. The SU-8 template's reflection peak is around $2.36 \mu \mathrm{m}$, whereas $\mathrm{ZnO}$ inverse sample's major reflection peak is around $3.67 \mu \mathrm{m}$. These two reflection peaks are in good agreement with the two pronounced dips in transmission. The reflection peak of the $\mathrm{ZnO}$ inverse sample is redshifted to that of the SU-8 template since the refractive index of the $\mathrm{ZnO}$ is higher than that of the SU-8. We also calculated the band structure by using the plane wave expansion method with the parameters deduced from Figs. 1(a) and 1(c). In the case of Fig. 1(a), the parameters are as follows: the lattice spacing is $1.21 \mu \mathrm{m}$ and the filling factor of the SU8 is $35.35 \%$. In the case of Fig. $1(\mathrm{c})$, the parameters are as follows: the lattice spacing is $1.23 \mu \mathrm{m}$ and the filling factor of the $\mathrm{ZnO}$ is $67.50 \%$ (the difference of the lattice spacing is due to the annealing of $\mathrm{ZnO}$ ). The refractive index for $\mathrm{SU}-8$ and $\mathrm{ZnO}$ were chosen to be 1.6 and 2.0, respectively. The band structures of SU-8 template and $\mathrm{ZnO}$ inverse samples are shown in the Figs. 2(b) and 2(c), respectively.

From the measured and calculated results shown in Fig. 2 , it can be observed that the position of the major peak in reflection corresponds well with the first partial stop band (outlined by the gray shaded square) expected from the band structure calculation for the measured direction. Where, the
$\Gamma-T$ is [ $\left.\begin{array}{lll}1 & 1 & 1\end{array}\right]$ direction of the fabricated fcc-type PCs, which is the measured direction. There are two shoulders in the reflection spectra of the $\mathrm{ZnO}$ infiltration structure. The first one at $2.60 \mu \mathrm{m}$ is due to the Fabry-Perot effect of the thin film and the second one at $1.92 \mu \mathrm{m}$ is good corresponding to the second partial stop band [outlined by the gray shaded square in Fig. 2(c)].

Figure 3(a) shows the photoluminescence (PL) spectra of the $\mathrm{ZnO} \mathrm{PC}$ and the bulklike porous $\mathrm{ZnO}$ fabricated on the same substrate and at the same time but outside the area with $\mathrm{PC}$ structure, which we will refer to as the reference material. The PL peak of the $\mathrm{ZnO}$ PC blueshifted to about $378.8 \mathrm{~nm}$, which is corresponding to the transmission dip of this sample. The transmission of the reference $\mathrm{ZnO}$ showing typical transmission step at band edge for bulk $\mathrm{ZnO}$. Furthermore, band edge exciton is five times more intense for $\mathrm{ZnO}$ PC compared to nonstructured PC under the same $\mathrm{cw}$ laser $(\mathrm{He}-\mathrm{Cd}$ laser at $325 \mathrm{~nm}$ ) excitation condition [see Fig. 3(b) inset]. This result clearly shows the PC structure strongly enhanced the exciton UV emission in $\mathrm{ZnO}$. Furthermore, peak intensity ratio of band edge exciton peak to the impurity/defect emission peak $(580 \mathrm{~nm})$ is about 1:1 for the $\mathrm{ZnO} \mathrm{PC}$, whereas this ratio drops to 1:6 for the nonstructured $\mathrm{ZnO}$ region. We also observed a difference in the exciton PL lifetime $(\tau)$ obtained from the time-resolved PL measurements (by fitting the curves to a single exponential decay model). As shown in Fig. 3(b), $\mathrm{ZnO}$ PC exhibited $\tau$ of $\sim 34$ ps, which is much shorter than that of the reference $\mathrm{ZnO}$ (about $88 \mathrm{ps}$ ). Reduction in the $\mathrm{ZnO}$ PC PL lifetime to $38.6 \%$ of that of the reference $\mathrm{ZnO}$ indicates that the partial photonic band gap of the structure has enhanced the spontaneous emission rate of $\mathrm{ZnO}$. It is known that a photon inhibition takes place if an electronic transition wavelength overlaps with the photonic band gap, and the transition is limited by the photon density of state (DOS). In this situation, the transition probability $p$ varies as the photon DOS, $p=1 / \tau$ $\propto$ DOS. So, the decrease in the lifetime is likely due to the increase in the photon DOS of the photon at the higher order photonic band edge. Recently, Cao and co-workers ${ }^{16}$ have 

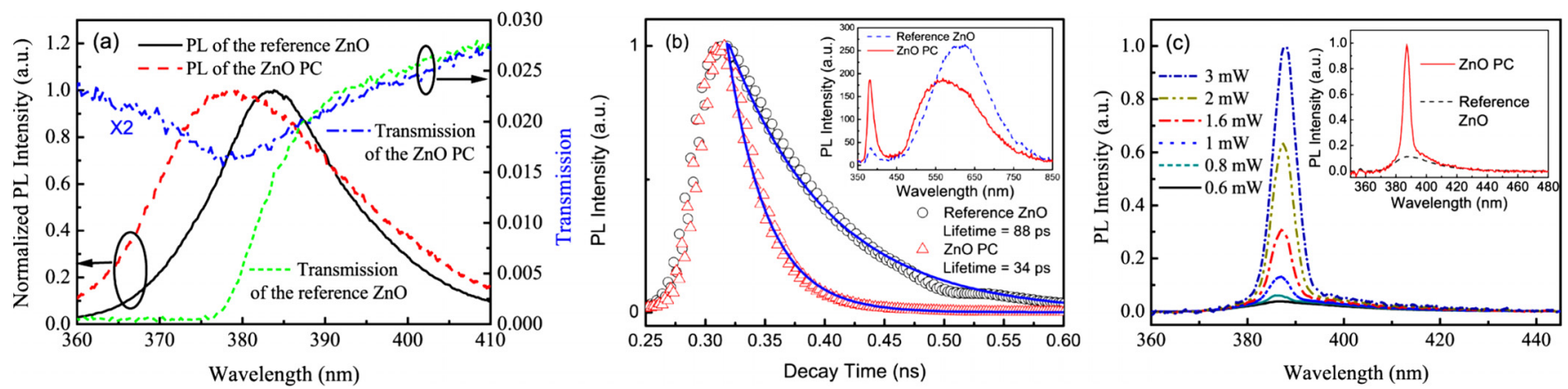

FIG. 3. (Color online) (a) PL and transmission spectra of the $\mathrm{ZnO}$ PC and the reference $\mathrm{ZnO}$. (b) Time-resolved measurement of the reference $\mathrm{ZnO}$ (black circle) and the $\mathrm{ZnO}$ with PC structure (red triangle). The blue solid lines are single exponential fitting curve of the data. The figure inset shows the comparison of the exciton emission intensity of the reference $\mathrm{ZnO}$ (red solid curve) and the $\mathrm{ZnO}$ with PC structure (blue dashed curve) under the same excitation condition. (c) Emission spectrum as a function for excitation power for the ZnO PC. The figure inset shows the ASE/PL spectra for ZnO PC and nonstructured $\mathrm{ZnO}$ at the same excitation intensity of $6.4 \mathrm{~mJ} / \mathrm{cm}^{2}$.

indeed shown that spontaneous emission is strongly modified by $\mathrm{DOS}$ for $\mathrm{ZnO}$ inverse opal structure. The enhanced spontaneous emission rate also manifested in significantly reduced threshold for the amplified spontaneous emission (ASE) in the PC $\mathrm{ZnO}$ compared to reference material. Figure 3(c) displays the emission spectra of the $\mathrm{PC} \mathrm{ZnO}$ as a function of excitation intensity. A sharp emission peak appears on top of the broad spontaneous emission band at an excitation threshold of $\sim 4 \mathrm{~mJ} / \mathrm{cm}^{2}$ (corresponded to $\sim 1 \mathrm{~mW}$ excitation power). A nonlinear increase in the emission intensity as a function of pumping energy and a corresponding reduction in emission spectral width (full width at half maximum) above the ASE threshold is also clearly seen. The inset of Fig. 3(c) shows the emission spectra of $\mathrm{ZnO}$ with and without the PC structure at the same excitation intensity, sharp ASE peak is clearly seen for the $\mathrm{ZnO}$ with fcc-type structure, while nonstructured reference $\mathrm{ZnO}$ only shows the broad spontaneous emission band. It is well known that $\mathrm{ZnO}$ in various forms can easily produce lasing effect under moderate optical excitation intensity. ${ }^{17-19}$ Indeed, both of the electrodeposited $\mathrm{ZnO} \mathrm{PC}$ and nonstructured $\mathrm{ZnO}$ can produce ASE but the ASE threshold for the PC $\mathrm{ZnO}$ is $\sim 4 \mathrm{~mJ} / \mathrm{cm}^{2}$ which is about three times smaller than the nonstructured $\mathrm{ZnO}$, in agreement with previous report on the reduction in lasing threshold in $\mathrm{ZnO}$ PC. ${ }^{12}$

To summarize, the infiltration of $\mathrm{ZnO}$ using electrodeposition into the PC polymer templates prepared by HL technique has been achieved. Complete filling of the narrow pores without any discontinuity is seen. Clear enhancement of spontaneous emission and lowering of the ASE threshold is seen for the $\mathrm{ZnO}$ with PC structure.

This work is partially supported by the Research Grants Council of Hong Kong (Project No. 603908) and the Univer- sity Development Fund and the Small Project Grant of the University of Hong Kong and the Fundamental Research Funds for the Central Universities (Grand No. 21609604).

${ }^{1}$ E. Yablonovitch, Phys. Rev. Lett. 58, 2059 (1987).

${ }^{2}$ Y. A. Vlasov, X. Z. Bo, J. C. Sturm, and D. J. Norris, Nature (London) 414, 289 (2001).

${ }^{3}$ H. B. Sun, S. Matsuo, and H. Misawa, Appl. Phys. Lett. 74, 786 (1999).

${ }^{4}$ M. Campbell, D. N. Sharp, M. T. Harrison, R. G. Denning, and A. J. Turberfield, Nature (London) 404, 53 (2000).

${ }^{5}$ B. H. Juárez, P. D. García, D. Golmayo, A. Blanco, and C. López, Adv. Mater. 17, 2761 (2005)

${ }^{6}$ M. Scharrer, X. Wu, A. Yamilov, H. Cao, and R. P. H. Chang, Appl. Phys. Lett. 86, 151113 (2005).

${ }^{7}$ J. H. Moon, S. Yang, W. Dong, J. W. Perry, A. Adibi, and S. M. Yang, Opt. Express 14, 6297 (2006).

${ }^{8}$ J. H. Moon, Y. Xu, Y. Dan, S. M. Yang, A. T. Johnson, and S. Yang, Adv. Mater. 19, 1510 (2007)

${ }^{9}$ M. Wang and X. Wang, Sol. Energy Mater. Sol. Cells 92, 357 (2008).

${ }^{10}$ X. Yu, Y. J. Lee, R. Furstenberg, J. O. White, and P. V. Braun, Adv. Mater 19, 1689 (2007).

${ }^{11}$ Ü. Özgür, Y. I. Alivov, C. Liu, A. Teke, M. A. Reshchikov, S. Doğan, V. Avrutin, S. J. Cho, and H. Morkoç, J. Appl. Phys. 98, 041301 (2005).

${ }^{12}$ L. K. Teh, C. C. Wong, H. Y. Yang, S. P. Lau, and S. F. Yu, Appl. Phys. Lett. 91, 161116 (2007).

${ }^{13}$ L. Wu, Y. C. Zhong, C. T. Chan, K. S. Wong, and G. P. Wang, Appl. Phys. Lett. 86, 241102 (2005).

${ }^{14}$ L. Z. Cai, X. L. Yang, Q. Liu, and Y. R. Wang, Opt. Commun. 224, 243 (2003).

${ }^{15}$ Y. Zhong, J. Zhou, and K. S. Wong, J. Appl. Phys. 107, 074311 (2010).

${ }^{16}$ H. Noh, M. Scharrer, M. A. Anderson, R. P. H. Chang, and H. Cao, Phys. Rev. B 77, 115136 (2008).

${ }^{17}$ M. H. Huang, S. Mao, H. Feick, H. Yan, Y. Wu, H. Kind, E. Weber, R. Russo, and P. Yang, Science 292, 1897 (2001).

${ }^{18}$ W. M. Kwok, A. B. Djurišić, Y. H. Leung, W. K. Chan, and D. L. Philips, Appl. Phys. Lett. 87, 223111 (2005).

${ }^{19}$ Z. Qiu, K. S. Wong, M. Wu, W. Lin, and H. Xu, Appl. Phys. Lett. 84, 2739 (2004). 